\title{
On control of flow instabilities in cutting of metals
}

\author{
Dinakar Sagapurama, Ho Yeunga, Yang Guob, Anirban Mahatoa, Rachid M’Saoubi (1)c, W. Dale Comptona, Kevin P. \\ Trumblea, Srinivasan Chandrasekara
}

a Center for Materials Processing and Tribology, Purdue University, West Lafayette, IN, USA

b M4 Sciences LLC, West Lafayette, IN, USA

c Seco Tools (UK) Ltd., Springfield Business Park, Alcester, UK

Large strain plastic flow in cutting of metals is studied at multiple length scales using high-speed imaging and marker techniques, complemented by particle image velocimetry and electron microscopy. Quantitative analysis of streak-lines, strain fields and microstructure, shows the flow to be often unsteady. Instabilities such as segmentation driven by ductile fracture, vortex-like flow in ductile metals, and shear banding in low-thermal diffusivity systems are elucidated using direct observations. A constrained-cutting process is demonstrated for suppressing the instabilities and unsteady flow.

Machining, Deformation, High-speed imaging

\section{Introduction}

Plastic deformation at small length scales on surfaces is intrinsic to machining processes, sliding, and surface deformation processing. While surface generation in these processes occurs by cumulative action of tools, the loading of a single indenter against the surface captures key aspects of the mechanics of material removal and deformation. For these reasons, it is a model system for studying flow phenomena underlying material removal.

The near-surface plastic flow determines how material is separated from the workpiece (WP), as well as characteristics of the newly generated surface such as microstructure, strength and residual stress. Unique features of this flow are imposition of large strains (1-10); its unconfined nature, unlike in bulk deformation processing; and a deformation zone that is on the length scale of the grain size. It has long been recognized, more so theoretically, that the lack of flow confinement can result in plastic instabilities and non-uniqueness in flow modes. The diverse variety of chip-particles and surface patterns produced in cutting, e.g., discrete particles, segmented chips, wrinkled particle surfaces, bear testimony to this lack of confinement. Their occurrence has been attributed to various mechanisms adiabatic shear localization [1], grain-level inhomogeneity of flow, local fracture [2, 3], and bifurcative instability [4] operative in the primary deformation zone. But these mechanisms have been largely analyzed in the framework of steady (smooth) flows and simplified velocity fields using upper bound methods, slip line fields (SLFs) and finite element analysis (FEA) [5]. The unconfined surface flow and its unique mesoscale characteristics, however, point to unsteady flows playing a critical role in triggering flow instabilities and localization, and creation of the particle morphologies and surface patterns. Important clues in this regard have emerged recently from direct in situ characterization of sliding and cutting at high spatial and temporal resolution [6-8].

This paper describes mesoscale characteristics of flows in cutting using high-speed image analyses, with emphasis on flow instabilities and their role in chip formation and surface generation. Possible control of unsteady flows and instabilities via design of deformation geometry is also discussed.

\section{Experimental}

Plane-strain cutting experiments were carried out in linear (planing, low-speed, HSS tool) and rotary (radial plunge turning, high-speed, carbide tool) cutting configurations. The tool rake angle $(\gamma)$ was set in the range of $+15^{\circ}$ to $-50^{\circ}$. Figure 1 shows the linear cutting wherein the chip formation was observed using a high-speed camera (pco dimax) coupled to an optical microscope at framing rates of 1-40 kHz. Quantitative flow parameters, e.g., strain, strain rate, streak-lines, were obtained by particle image velocimetry (PIV) analysis of the images [6, 8]. A marker technique was used to analyze flow in high-speed cutting $\left(v_{c} \sim\right.$ $0.25-5 \mathrm{~m} / \mathrm{s})$. Finely-spaced $(\sim 5 \mu \mathrm{m})$ parallel markers were machined onto one side of a disk WP prior to the cutting [9]. This side-surface was constrained to ensure plane strain. The WP materials, selected to illustrate specific instabilities, were: halfhardened single-phase brass 260 and $\mathrm{Al} 1100$, representing partially work-hardened metals; Mg AZ31B as a model system for poor-workability alloys; annealed OFHC $\mathrm{Cu}$ for highly ductile metals; and Ti-6Al-4V, representing hard metals with low thermal diffusivity. A small set of cutting experiments was also carried out wherein the final chip thickness $\left(t_{c}\right)$ was controlled by placing an additional constraint directly across from the cutting edge at the point of chip formation, as shown in Fig. 1. Details of this constrained cutting may be found in Ref. [10].
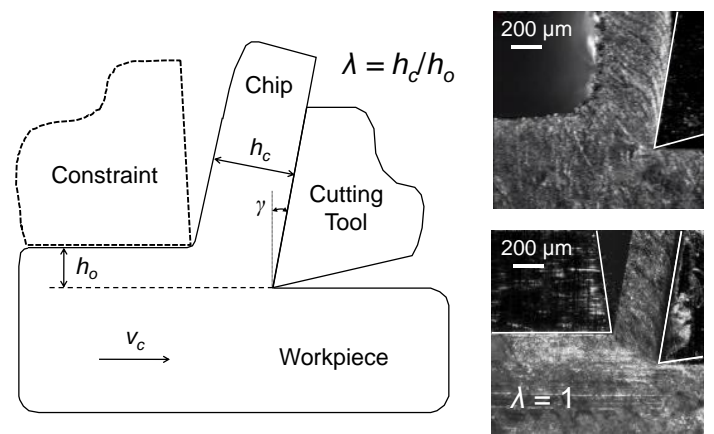

Figure 1. Linear cutting arrangement. Images show flow in conventional (top) and constrained (bottom) cutting. WP width was $2 \mathrm{~mm}$. 


\section{Segmentation instability (partially work-hardened metals)}

Figure 2 shows select frames, with superimposed (virtual) grid, from a high-speed sequence of cutting of brass $260\left(\gamma=-50^{\circ}\right)$, highlighting a flow instability that triggers chip segmentation. The alpha brass had a grain size of $\sim 15 \mu \mathrm{m}$, Vickers hardness $\sim 137 \mathrm{~kg} / \mathrm{mm}^{2}$, and WP roughness $\mathrm{Ra} \sim 0.1 \mu \mathrm{m}$. A prow (A) of material develops ahead of the tool. But the flow is unsteady and the prow unstable, as seen in the sequence. A crack initiates on the prow surface (red arrow, frame 1), triggering a surface flow instability, and propagates towards the tool tip (frames 1-3, yellow arrow marks crack tip). When it reaches the tip, prow $A$ is separated from the WP as a discrete chip (fully segmented, frame 3 ). The crack propagates at $\sim 0.7 \mathrm{~mm} / \mathrm{s}$ initially, slowing to $\sim 0.5$ $\mathrm{mm} / \mathrm{s}$ as it approaches the tool. This speed is much lower than adiabatic shear failure speeds but quite similar to the cutting speed of $1 \mathrm{~mm} / \mathrm{s}$, indicating a stable growth of the crack, drivenin by the advancing tool. The propagation of the crack is in a ductile mode. Simultaneously, a new prow develops below A (frames 2-4) until it is somewhat constrained by the tool (frame 4); material flow through the prow is now quickly retarded. A crack then initiates as before at the prow surface (red arrow, frame 4) even as A is squeezed out as a chip. The next cycle of discrete chip formation now begins. This discrete chip formation was also noted in partially-hardened $\mathrm{Cu}, \mathrm{Al}$ and Ti. Besides highlighting the unsteady surface flow, the observations show conclusively, that discontinuous chip formation and segmentation are due to a ductile crack originating on the free (back) surface of the chip. While the free surface failure initiation has been suggested in prior work $[3,11,12]$, mainly with hard (brittle) steels and $\mathrm{Ti}-6 \mathrm{Al}-4 \mathrm{~V}$, the present results are the first confirmation using in situ observation, including for cutting of ductile metals.
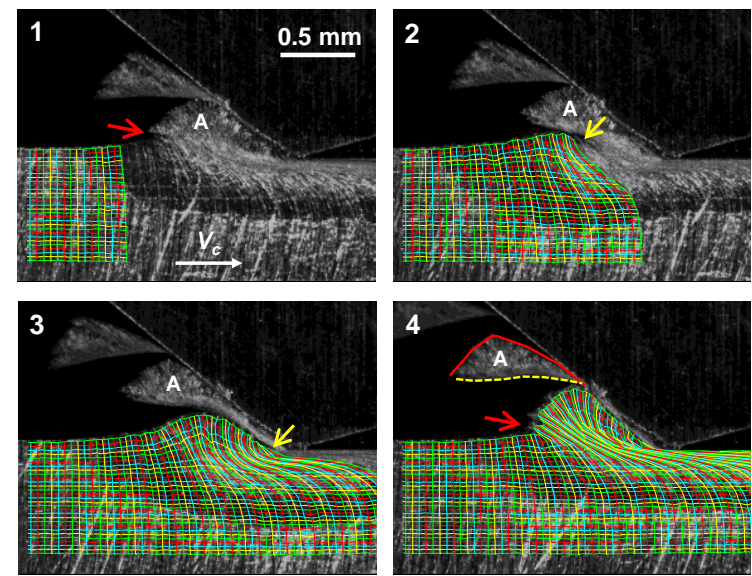

Figure 2. Flow evolution in segmented chip formation. Brass, $\gamma=-50^{\circ}, v_{c}=$ $1 \mathrm{~mm} / \mathrm{s}, h_{o}=50 \mu \mathrm{m}$, inter-frame $=0.8 \mathrm{~s}$.

When $\gamma$ is made less negative, the discrete chip transitioned to a segmented chip, with serrated pattern on its surface, due to the prow crack propagating only part way into the chip. At $\gamma=0^{\circ}$, a continuous chip resulted but with a free surface that was still rough $\left(\sim 0.08 h_{c}\right)$ at the mesoscale; this roughness was due to the prow cracks being arrested very soon after initiation. The in situ observations thus showed that these chip morphologies discrete, segmented and continuous with small-scale roughness all arise from the same, universal ductile failure operating at different scales. Interestingly, the prow slope at crack initiation $\left(\theta^{*}\right)$ was constant at $\sim 35^{\circ}$ and independent of $\gamma$ (see Fig. 3a for discontinuous chip). Similar segmentation, with $\theta^{*}$ of $30^{\circ}-35^{\circ}$, was also observed with $\mathrm{Al}$ (Fig. 3b) and $\mathrm{Ti}$, suggesting $\theta^{*}$ is determined primarily by the deformation geometry.
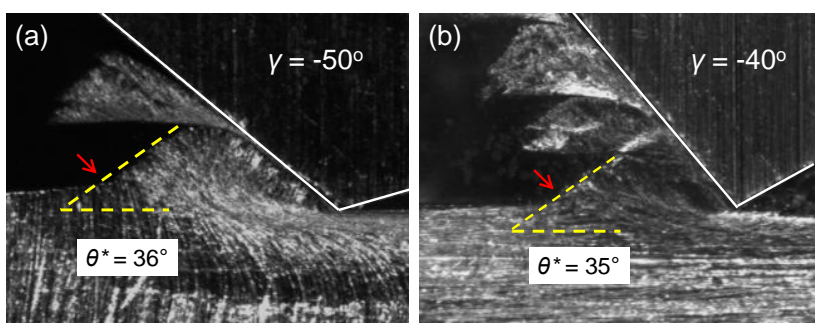

Figure 3. Prow slope at crack initiation (red arrows) in (a) brass 260 ( $\gamma=$ $\left.-50^{\circ}, v_{c}=1 \mathrm{~mm} / \mathrm{s}, h_{o}=50 \mu \mathrm{m}\right)$ and (b) Al $1100\left(\gamma=-40^{\circ}, v_{c}=5 \mathrm{~mm} / \mathrm{s}, h_{o}=\right.$ $50 \mu \mathrm{m})$. Note that $\theta^{*}$ is essentially the same for the two systems.

The segmentation also left its imprint on the machined surface in the form of a heterogeneous strain distribution (Fig. 4) along the cut length, due to the prow instability. The maximum strain at the surface varied between 4 and 2.5. The unsteady surface flow thus can have undesirable consequences for surface quality, beyond surface finish degradation [13] and force oscillations.

Segmentation is also quite pronounced in hexagonal closepacked (hcp) crystal structure metals like Mg alloys, where poor workability is due to limited slip activity at room temperature. This segmentation is reflected in a discrete chip macrostructure (Fig. 5a) and highly nonhomogeneous microstructure (Fig. 5b).

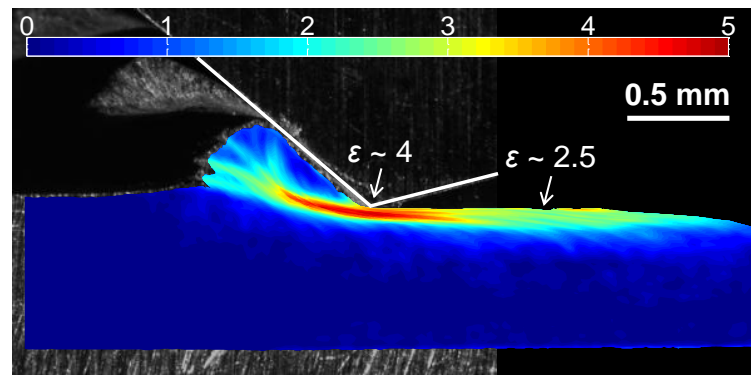

Figure 4. WP strain field heterogeneity in segmented chip formation. Brass 260, $\gamma=-50^{\circ}, v_{c}=1 \mathrm{~mm} / \mathrm{s}, h_{o}=50 \mu \mathrm{m}$.
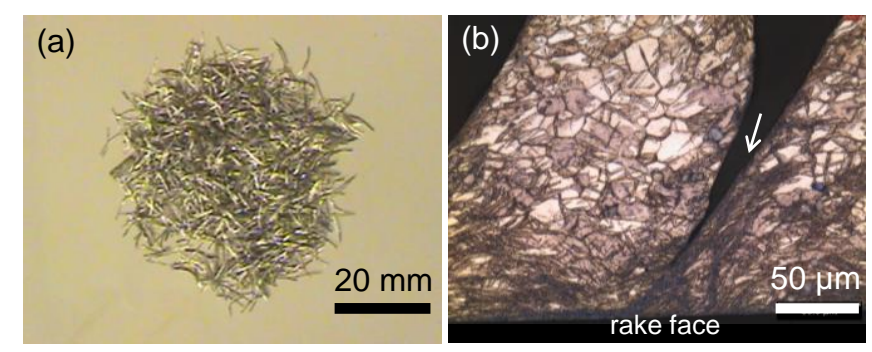

Figure 5. Segmented chip in Mg AZ31B characterized by (a) discrete needle-like macrostructure, and (b) nonhomogeneous microstructure. The rake face and chip-margin (arrow) exhibit dark etching contrast indicative of high-straining. $\gamma=5^{\circ}, v_{c}=1.2 \mathrm{~m} / \mathrm{s}, h_{o}=250 \mu \mathrm{m}$.

\section{Vortex-like flow and folding instability}

That cutting of soft annealed metals can pose difficulties has long been known in machining practice. The forces are often high and there is significant side flow of metal leading to the designation of poor machinability. The large forces have been attributed to a small shear angle [3]. To better understand these observations, the nature of flow in cutting of annealed $\mathrm{Cu}(\sim 500$ $\mu \mathrm{m}$ grain size) was explored using high-speed imaging.

Figure 6 shows two frames, with streak-lines superimposed, depicting the flow. A streak-line is locus of material particles that have previously passed through a particular spatial point. The flow is quite unusual characterized by highly sinuous streak-lines, 
significant rotation, and folding over of the lines throughout the chip akin to "rope-coil" flow in viscous liquids. This flow cannot be described in the framework of the usual cutting models. The arrows in the figures track the same material points as the chip forms. The sinuous flow with folding is triggered by small wrinkles that develop on the WP surface (see arrows, frame 1), reminiscent of a similar flow in sliding $[6,7]$. This unsteady flow is termed vortex-like, and the instability, a folding instability. This flow triggers self-contacts on the chip free surface, giving it a highly wrinkled morphology (see inset in Fig. 6).
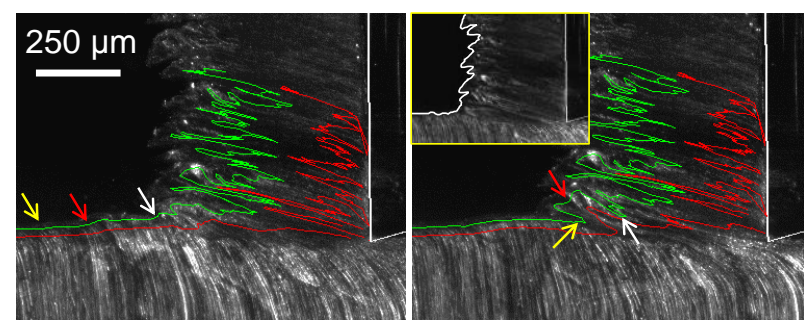

Figure 6. Image frames (inter-frame $0.3 \mathrm{sec}$ ) with streak-lines showing folding and highly sinuous flow in annealed $\mathrm{Cu}$. Wrinkled chip surface due to the instability is shown in inset. $\gamma=0^{\circ}, v_{c}=0.4 \mathrm{~mm} / \mathrm{s}, h_{o}=50 \mu \mathrm{m}$.

The reason for the poor machinability of annealed metals is now apparent. It is a consequence of the vortex-like flow involving extensive redundant deformation. The severity of the vortex-like flow (and forces) was found to decrease if the WP was strain hardened prior to the cutting. While the origin(s) of this flow is as yet unresolved, it likely involves WP microstructure heterogeneity and strain hardening capacity [6]. As with segmentation, the WP surface plays a key role in this instability.

\section{Shear banding}

Flow localizing by adiabatic shear is common in high-speed cutting of low-thermal diffusivity alloys such as $\mathrm{Ti}$ and $\mathrm{Ni}[1,2$, 13]. Figure 7 is a high-speed image sequence of this instability in Ti-6Al-4V. The Ti-6Al-4V, in an initial mill-annealed condition, had grain size of $3 \mu \mathrm{m}$ and hardness of $346 \mathrm{HV}$. The instability is seen to be a two-step process. The first step is creation of a planar "weak path", fully established by frame 3 in Fig. 7 (see at arrow), that precedes subsequent localized flow. The path development is rapid and occurs between frames 2 and 3, giving a lower-bound of $8 \mathrm{~m} / \mathrm{s}$ for the propagation front velocity. The second step is shear along the localized band (frames 4-6), with each side of the band sliding essentially as a rigid body. This step, resembling stack-of-cards shear, reduces the load-bearing area; this will

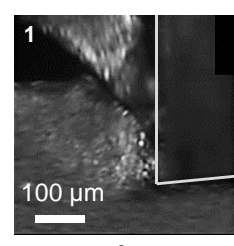

$\mathrm{t}=0 \mu \mathrm{s}$

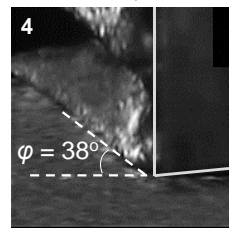

$\mathrm{t}=125 \mu \mathrm{s}$

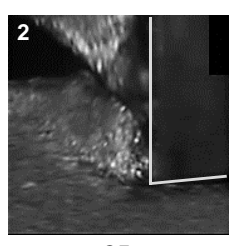

$t=25 \mu s$

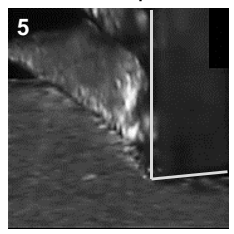

$t=200 \mu s$

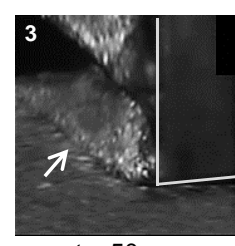

$\mathrm{t}=50 \mu \mathrm{s}$

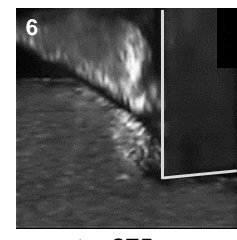

$\mathrm{t}=275 \mu \mathrm{s}$
Figure 7. Image sequence of shear band development in Ti-6Al-4V ( $\gamma=$ $\left.0^{\circ}, v_{c}=0.65 \mathrm{~m} / \mathrm{s}, h_{o}=200 \mu \mathrm{m}\right)$. Frames $1-3$ show initiation of a "weak path" (arrow), followed by shear (sliding) along this path in frames 4-6. contribute to flow softening in addition to the adiabatic conditions. The sliding velocity $(0.78 \mathrm{~m} / \mathrm{s})$ is close to the shear velocity $\left(v_{s} \sim 0.83 \mathrm{~m} / \mathrm{s}\right)$ estimated for the stack-of-cards model, as $v_{s}=\cos \gamma / \cos (\varphi-\gamma) v_{c}$, with $\varphi$ the shear angle (frame 4, Fig. 7).

The characteristics of the shear-banded chip, including displacement of specific marker lines used to compute local strain, are shown in Fig. 8. The "saw-tooth" shape and marker displacements are due to localized flow in the bands. These shear displacements (arrows, Fig. 8a) at various points along the band were essentially equal $(\delta \sim 80 \mu \mathrm{m})$ and similar to the shear offset at the surface. This implies uniform straining along the band. The band (local) shear strain $\left(\gamma_{s b}\right)$ is computed as the ratio of the marker displacement $(\delta)$ to band width $(\Delta)$. Measurements of $\Delta$ from at least 10 bands using optical, electron microscopy and diffraction methods yielded self-consistent results, and showed $\Delta$ to be strongly dependent on $v_{c}$ [9]. For $v_{c}=1 \mathrm{~m} / \mathrm{s}$ in Fig. 8, $\Delta$ was $3.9 \pm 0.6 \mu \mathrm{m}$, giving $\gamma_{s b}$ of 22.6. The corresponding local strain rate, $v_{s} / \Delta$, is $4 \times 10^{5} / \mathrm{s}$, at least an order higher than in continuous chip formation. The band strain ranged from $\gamma_{s b} \sim 10\left(v_{c}=0.25\right.$ $\mathrm{m} / \mathrm{s})$ to $\sim 40\left(v_{c}=5 \mathrm{~m} / \mathrm{s}\right)$, as in dynamic punching and testing.

The markers, equivalent of streak-lines, are useful also for estimating the strain in the regions adjoining the bands. The yellow line in Figure 8a highlights a marker across two chip segments and a band. This streak-line is curved at the band margins, revealing deformation gradients established due to the localized shear. The strain profile, obtained from the local streakline curvature, is shown in Fig. 8c. The uniform strain within the band $\left(\gamma_{s b}\right)$, obtained from measurements of $\delta$ and $\Delta$, is shown by a dotted line. The strain gradient is quite steep $(\sim 20 / \mu \mathrm{m})$ in the immediate vicinity of the band (within $1 \mu \mathrm{m}$ ), followed by a much gentler decay. This gradient is reflected in the microstructure (Fig. 8b), with the shear-banded region (arrows) showing highly distorted structure, and the less-deformed region between the bands preserving the original grain structure. Transmission electron microscopy (TEM) of the band showed nanoscale structures with $\sim 50-100 \mathrm{~nm}$ grain size (Fig. 9) - a consequence of the extreme deformation occurring in the band.

The image analysis and related characterization has shown that shear banding is a 2-step process, with a contribution to flow softening likely also resulting from load-bearing area changes during the shear. Local deformation parameters in the band and its vicinity can be measured, enabling correlations with microstructure and strength properties. The multi-scale characterization, besides being of value for validating machining models, can also provide insights into flow localization mechanisms and dynamic flow stress in the band.
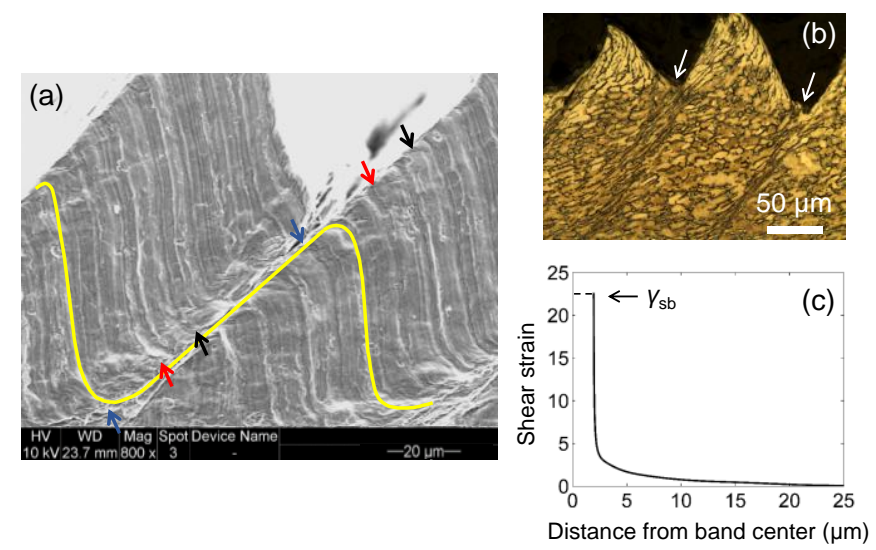

Figure 8. Shear banding in Ti-6Al-4V: (a) SEM image showing displacement of specific markers (arrows) due to localized shear, (b) optical micrograph of chip microstructure, and (c) strain profile in band vicinity. $\gamma=0^{\circ}, v_{c}=1 \mathrm{~m} / \mathrm{s}, h_{o}=125 \mu \mathrm{m}$. 


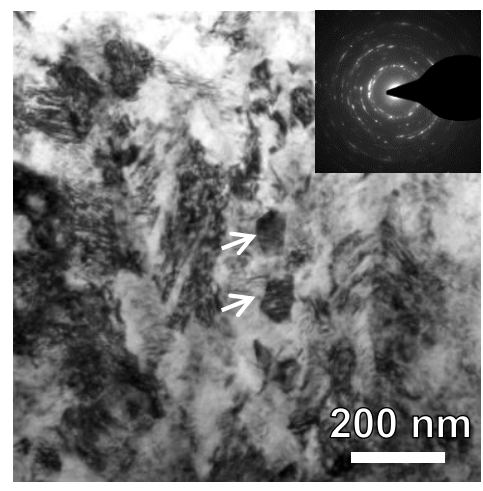

Figure 9. Bright-field TEM image and diffraction pattern showing nanocrystalline structure $(\sim 50-100 \mathrm{~nm}$ grains, arrows $)$ inside Ti-6Al-4V shear band.

\section{Suppressing flow instabilities}

The results have shown that unsteady flows and various instabilities in cutting are mediated by events on the WP free surface and initial WP state (e.g., hardening capacity, microstructure). In the case of segmentation, this impetus is prow formation followed by cracking; with the folding, it is formation of small wrinkles; and with the shear banding, it is accommodation of the unconfined macroscale shear displacement that also impacts flow softening. These observations suggest that if the chip formation could be modified by application of a suitable constraint in the deformation zone (Fig. 1), thereby, converting it into (constrained) hybrid cutting-deformation, then opportunities may exist for negating free surface effects and flow instabilities. Figures 10a and b demonstrate the successful outcome of this constrained cutting approach in controlling segmentation (Mg AZ31B) and shear banding (Ti-6Al-4V). The constraint level, set by the ratio $\lambda=h_{c} / h_{o}$, was 0.7 for $\mathrm{Mg}$ and 0.6 for Ti. Particular attention must be paid to the uniformity of the chip microstructure in these figures, for this is the unambiguous signature of suppression of instability. For reference, contrast these microstructures with the heterogeneous ones in Figs. 5b and $\mathbf{8 b}$ produced at similar cutting conditions, but without constraint. The occurrence of a continuous chip, while a necessary signature, is not a sufficient one for demonstrating lack of flow instability. The transformation of the discrete chips in conventional machining of Mg AZ31B (Fig. 5a) to a continuous foil of very long length in the constrained cutting (Fig. 10a) is quite remarkable. The constrained cutting has also been successful in suppressing the vortex-like flow in annealed metals.

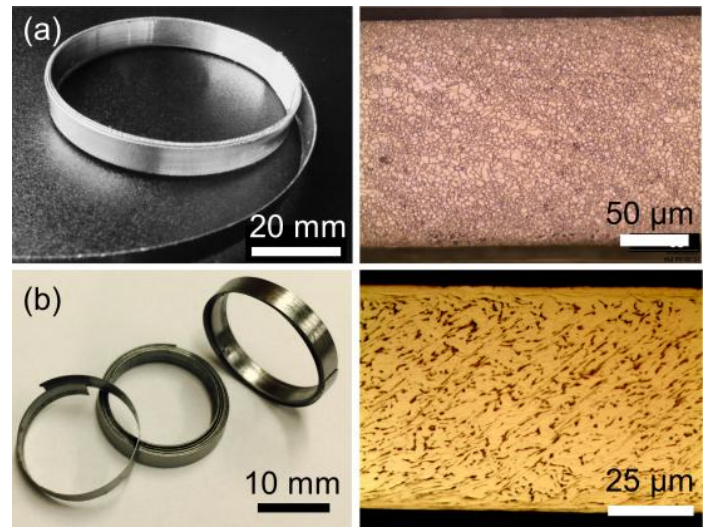

Figure 10. Suppression of flow instabilities by constrained cutting: continuous chip morphology (left) and the associated homogeneous microstructure (right) for (a) $\operatorname{Mg} \operatorname{AZ31B}\left(\lambda=0.7, \gamma=0^{\circ}, v_{c}=1 \mathrm{~m} / \mathrm{s}, h_{o}=\right.$ $250 \mu \mathrm{m})$ and (b) Ti-6Al-4V $\left(\lambda=0.6, \gamma=0^{\circ}, v_{c}=1 \mathrm{~m} / \mathrm{s}, h_{o}=125 \mu \mathrm{m}\right)$.
It must be noted that suppression of the free surface effects requires a constraint of sufficiently small $\lambda$, and not just any $\lambda$. This $\lambda$ value can be estimated a priori by assessing the surface displacements that trigger/accommodate the flow instability: with segmentation, this is related to prow height; for shear banding, by the shear offset; and in the case of the vortex-like flow, by the wrinkling. The constrained machining has another key characteristic - confinement of the plastic-work induced heating that promotes near-adiabatic conditions in the deformation zone - of extraordinary value for enhancing slip in hcp alloys, and production of metal sheet with fine-grained microstructures and shear-textures by chip formation [14].

The observations also suggest other approaches to suppression of instabilities such as by control of the loading cycle (e.g., modulation-assisted machining), and initial WP texture. Preliminary results have shown promise in the former regard.

\section{Concluding remarks}

The flow characteristics in cutting of diverse metal systems have been studied using in situ image analysis. The flow is unsteady and at variance with the usual models of cutting. The development of three types of flow instabilities are highlighted, namely segmentation, vortex-like flow with folding, and shear banding. The work surface plays a major role in triggering the instabilities. By application of a constraint of sufficient level in the deformation zone and modifying the surface boundary conditions, suppression of unsteady flow and flow instabilities is demonstrated. This can enhance workpiece quality by ensuring a uniform deformation state on the machined surface.

\section{Acknowledgement}

This work was supported by U.S. DOE DE-EE0005762 (via Third Wave Systems), NSF CMMI 1363524 and 1234961, and U.S. ARO W911NF-12-1-0012.

\section{References}

[1] Komanduri R, Von Turkovich BF (1981) New Observations on the Mechanism of Chip formation when Machining Titanium Alloys. Wear 69:179-188.

[2] Shaw MC, Vyas A (1993) Chip Formation in the Machining of Hardened Steel. CIRP Annals-Manufacturing Technology 42:29-33.

[3] Nakayama K, (1974) The Formation of "Saw-Toothed Chip" in Metal Cutting. Proceedings of the International Conference on Production Engineering 1:572-577.

[4] Burns T], Davies MA (1997) Nonlinear Dynamics Model for Chip Segmentation in Machining. Physical Review Letters 79:447-450.

[5] Oxley PLB (1989) Mechanics of Machining: An Analytical Approach to Assessing Machinability. Ellis Horwood Publisher.

[6] Sundaram NK, Guo Y, Chandrasekar S (2012) Mesoscale Folding, Instability, and Disruption of Laminar Flow in Metal Surfaces. Physical Review Letters 109:106001.

[7] Mahato A, Guo Y, Sundaram NK, Chandrasekar S (2014) Surface Folding in Metals: A Mechanism for Delamination Wear in Sliding. Proceedings of the Royal Society A 470:20140297.

[8] Guo Y, M'Saoubi R, Chandrasekar S (2011) Control of Deformation Levels on Machined Surfaces. CIRP Annals-Manufacturing Technology 60:137-140.

[9] Sagapuram D, M'Saoubi R, Trumble KP, Chandrasekar S (2014) Shear Band Width, Strain and Microstructure in High-Velocity Shear Deformation. 17th U.S National Congress on Theoretical and Applied Mechanics paper: S-03-705.

[10] Moscoso W, Shankar MR, Mann JB, Compton WD, Chandrasekar S (2007) Bulk Nanostructured Materials by Large Strain Extrusion Machining. Journal of Materials Research 22:201-205.

[11] Usui E, Obikawa T, Shirakashi T (1984) Study on Chip Segmentation in Machining Titanium Alloy. Proceedings of the $5^{\text {th }}$ International Conference on Production Engineering pp. 233-239.

[12] Tonshoff HK, Arendt C, Ben Amor R (2000) Cutting of Hardened Steel. CIRP Annals-Manufacturing Technology 49:547-566.

[13] Davies MA, Burns TJ, Evans CJ (1997) On the Dynamics of Chip Formation in Machining Hard Metals. CIRP Annals-Manufacturing Technology 46:25-30.

[14] Sagapuram D, Efe M, Moscoso W, Chandrasekar S, Trumble KP (2013) Controlling Texture in Magnesium Alloy Sheet by Shear-Based Deformation Processing. Acta Materialia 61:6843-6856. 